\title{
Jean-Paul Willaime, Céline Béraud, (dirs), Les Jeunes, l'école et la religion
}

Paris, Bayard, 2009

\section{Clémentine Vivarelli}

\section{(2) OpenEdition}

\section{Journals}

\section{Édition électronique}

URL : http://journals.openedition.org/assr/22100

DOI : $10.4000 /$ assr. 22100

ISSN : $1777-5825$

\section{Éditeur}

Éditions de l'EHESS

Édition imprimée

Date de publication : 31 décembre 2010

Pagination : 9-242

ISBN : 9782713223013

ISSN : 0335-5985

\section{Référence électronique}

Clémentine Vivarelli, « Jean-Paul Willaime, Céline Béraud, (dirs), Les Jeunes, l'école et la religion »,

Archives de sciences sociales des religions [En ligne], 152 | octobre-décembre 2010, document 152-120, mis en ligne le 06 mai 2011, consulté le 21 septembre 2020. URL : http://journals.openedition.org/ assr/22100; DOI : https://doi.org/10.4000/assr.22100

Ce document a été généré automatiquement le 21 septembre 2020

(c) Archives de sciences sociales des religions 


\section{Jean-Paul Willaime, Céline Béraud, (dirs), Les Jeunes, l'école et la religion}

Paris, Bayard, 2009

\section{Clémentine Vivarelli}

\section{RÉFÉRENCE}

Jean-Paul Willaime, Céline Béraud, (dirs), Les Jeunes, l'école et la religion, Paris, Bayard, 2009

1 S'inscrivant plus largement dans le cadre d'une enquête réalisée sur le thème de l'école et de la religion en Europe, financée par l'Union Européenne et intitulée «REDCO » ( La religion dans l'enseignement : contribution au dialogue ou facteur de conflit dans les sociétés européennes en mutation?), le présent ouvrage collectif s'intéresse essentiellement au cas français.

2 Prenant pour point d'appui une enquête sociologique qualitative (cent trois entretiens réalisés dans huit établissements scolaires de Paris et banlieue, Metz et Rennes) et quantitative (huit cent cinquante et un questionnaires diffusés), les auteurs de cet ouvrage se sont intéressés à la fois aux expériences de professeurs ainsi qu'à celles de jeunes adolescents âgés de quatorze à seize ans.

3 Après avoir brièvement fait état des grandes étapes de l'histoire de la laïcité en France et rappelé ainsi la spécificité du cas français par rapport aux autres nations européennes, cet ouvrage retrace les conditions d'émergence du débat sur l'inculture des jeunes depuis les années quatre-vingts, et souligne par conséquent la nécessité d'y remédier par le biais d'un enseignement des faits religieux à l'école.

4 Développant une approche interdisciplinaire qualifiée d' "interpretativ approach ", cette enquête sociologique se focalise tout d'abord sur les représentations de la religion 
et de la laïcité chez les jeunes adolescents. Elle révèle alors une islamisation de la représentation de la religion, notamment reliée à la médiatisation intensive de la religion musulmane depuis le 11 septembre 2001, à une visibilité grandissante de celleci dans l'espace public, aux inquiétudes plus générales liées au terrorisme, etc. Bien que les jeunes aient majoritairement intériorisé une appréhension pluraliste de la religion, l'enquête fait pourtant état d'une représentation ethnicisée de la religion chez les jeunes français. Si l'on note encore l'impact persistant des appartenances traditionnelles liées à la socialisation familiale dans les phénomènes de croyances et d'affiliations religieuses, le thème de la religion reste malgré tout marginal dans la culture juvénile.

5 Les jeunes français semblent pourtant moins attachés à l'esprit athéiste que leurs parents, plus ouverts à d'autres formes de spiritualité, et défendent les valeurs du pluralisme et de la tolérance vis-à-vis de la religion de l'Autre de façon consensuelle. En ce qui concerne enfin leur représentation de la laïcité, l'enquête montre que les jeunes français privilégient avant tout une laïcité de voisinage, de proximité, vécue et pratiquée au quotidien, ne voyant pas nécessairement la nécessité d'une forme d'interventionnisme étatique. Jean-Paul Willaime évoque une banalisation du principe de laïcité dans le milieu scolaire, qui démontre ainsi les capacités de transmission de l'appareil scolaire, des valeurs de respect, de tolérance et d'une laïcité d'intelligence chez les jeunes, relativisant ainsi les discours alarmistes sur la crise de l'institution scolaire.

6 Si les jeunes reconnaissent de façon unanime l'utilité d'un enseignement des faits religieux abordés en cours d'histoire, de français et d'arts plastiques, l'enquête souligne la dimension avant tout patrimoniale de cet enseignement, au détriment d'une approche anthropologique basée sur l'expérience du religieux. Si la salle de classe semble constituer un lieu privilégié d'échange des opinions en raison de la diversité qui y est présente, les élèves font malgré tout part des difficultés à communiquer leur expérience religieuse personnelle et abordent ainsi bien plus facilement la question du rôle social des religions.

7 Le présent ouvrage se base ensuite sur des observations réalisées en classe, ainsi que sur les discours de professeurs, pour démontrer l'hétérogénéité des manières d'aborder le religieux dans l'enseignement. Prenant appui sur le savoir des élèves ou évitant à l'inverse d'y faire référence par crainte des conflits, les observations menées démontrent néanmoins l'existence d'un espace de dialogue majoritairement libre, où le thème de la religion permet de délivrer un message civique fort, d'éduquer à la tolérance et où la pédagogie du dialogue permet de surmonter les conflits.

8 L'enquête aborde, en dernier lieu, le domaine de l'enseignement privé et souligne de nombreuses disparités entre l'enseignement privé catholique et juif. Elle note globalement un phénomène de sécularisation du privé catholique malgré l'hétérogénéité de l'enseignement privé en France. Le monde de l'enseignement privé catholique reste malgré tout nettement plus conservateur que le domaine public, moins concerné par les défis du multiculturalisme puisque moins confronté à la pluralité en général, et moins favorable à la privatisation du religieux. L'analyse sociologique de l'enseignement privé juif fait au contraire état d'un phénomène de regroupement communautaire progressif. En relation avec des croyances et des pratiques religieuses fortes, les familles juives choisissent majoritairement de scolariser leurs enfants dans les écoles juives privées (plus d'un élève français de confession juive sur deux fréquente 
ce type d'établissement scolaire). Ce fort sentiment identitaire basé sur l'appartenance religieuse, le respect des interdits alimentaires et des fêtes religieuses juives, coexistent néanmoins avec les valeurs du pluralisme et même avec une certaine hostilité à l'égard des manifestations trop visibles du religieux au sein de l'espace scolaire (présence de lieux de prière et port de signes religieux).

9 L'enquête réalisée dans le cadre du projet REDCO a ainsi pour originalité de croiser la sociologie de la religion et la sociologie du milieu scolaire. Elle relativise les discours souvent alarmistes évoqués dans les différents rapports ministériels français, notamment le rapport Stasi, en soulignant l'intégration des valeurs du pluralisme et de la tolérance chez les jeunes. Elle contrebalance les discours faisant état de la crise de l'institution scolaire en apportant un regard basé sur un travail de terrain solide. En s'intéressant de façon novatrice au point de vue des adolescents, elle permet d'apporter un angle de compréhension nouveau sur les rapports entre religion et école, relativisant ainsi les discours sur l'inculture religieuse des jeunes. 